\title{
Effects of CA3 bursts on CA1 activity examined through a simple CA3-CA1 spiking network
}

\author{
loannis Taxidis ${ }^{1 *}$, Markus Owen ${ }^{1}$, Steve Coombes ${ }^{1}$, Robert Mason ${ }^{2}$ \\ From Nineteenth Annual Computational Neuroscience Meeting: CNS*2010 \\ San Antonio, TX, USA. 24-30 July 2010
}

The hippocampus and specifically the CA3-CA1 areas exhibit a variety of intrinsic rhythms that span frequencies from the slow theta range $(4-8 \mathrm{~Hz})$ up to fast ripples $(150-200 \mathrm{~Hz})$. Various computational models of different complexities have been developed in an effort to simulate such population oscillations and uncover their underlying mechanisms. Nevertheless most studies focus on specific rhythms generated in localized areas. They do not address more complicated phenomena such as the so called Sharp Wave-Ripple complex observed in CA1 EEG recordings, which is believed to be generated through the combination of large pyramidal dendritic depolarizations and fast synchronous interneuronal firing, induced by $\mathrm{CA} 3$ population bursts.

Here we present the combination of two simple but realistic models of CA3 and CA1 respectively, connected together in an effort to examine the effects of such CA3 bursts on CA1. Both network models are computationally simple one dimensional arrays of pyramidal and interneuron populations interacting only via fast AMPA and $\mathrm{GABA}_{\mathrm{A}}$ synapses. They were constructed using the 2-compartment Pinsky-Rinzel model for single pyramidal cells and the one-compartment Wang-Buzsaki model for interneurons, reproducing the basic firing properties of hippocampal cells. Connectivity schemes and postsynaptic potentials are based on biological data, making the network topology as realistic as possible.

Our CA3 model is a highly recurrent network that reproduces a number of different features observed in real recordings or simulations of more sophisticated models (e.g. activity propagation in the disinhibited array, Carbachol-induced oscillations (5-6 Hz) and others). Most importantly it exhibits semi-synchronised population bursts that have been observed in CA3 slices and in vivo.

Recurrent pyramidal cell connections are almost absent in the CA1 network which is based mostly on the interaction between pyramidal cells with a strongly connected interneuronal network. The main rhythm reproduced here is the gamma frequency of the pure interneuron population and its long range synchronization through the interaction with pyramidal cells. The two models are coupled together in a feedforward CA3to-CA1 scheme that mimics Shaffer collaterals.

The effects of CA3 bursts in the intrinsic CA1 activity are examined along with the role of various parameters values and stimuli. Our final goal is to simulate Sharp Wave-Ripple complexes and uncover the hippocampal features that generate them. We believe that our model is ideally suited to shedding further light on the potential mechanisms of such synchronous hippocampal events.

\section{Author details}

${ }^{1}$ School of Mathematical Sciences, University of Nottingham, Nottingham, NG7 2RD, UK. ' ${ }^{2}$ of Biomedical Sciences, University of Nottingham, Medical School, Nottingham NG7 2UH, UK.

Published: 20 July 2010

doi:10.1186/1471-2202-11-S1-P16

Cite this article as: Taxidis et al:: Effects of CA3 bursts on CA1 activity examined through a simple CA3-CA1 spiking network. BMC

Neuroscience 2010 11(Suppl 1):P16.

\footnotetext{
* Correspondence: pmxit@nottingham.ac.uk

${ }^{1}$ School of Mathematical Sciences, University of Nottingham, Nottingham, NG7 2RD, UK
} 